\title{
INVASIVE LOBULAR CARCINOMA OF THE BREAST: A CHALLENGE TO THE RADIOLOGIST
}

\author{
Wijegunawardhana D, Gunawardena D S \\ Department of Imaging Services, Royal Perth Hospital, Western Australia
}

\begin{abstract}
Invasive lobular carcinoma (ILC) is the second most common histological sub type of breast cancer among women. Its' characteristic immuno-histopathology leads to less conspicuous clinical and radiological features resulting in delayed diagnosis. The difference between ILC and invasive ductal carcinoma (IDC) with respect to risk factors, clinical features, imaging appearances and metastases etc. has been overlooked for many years. In this article, we attempt to review the available literature on ILC emphasizing the difference from IDC.
\end{abstract}

Keywords: Invasive lobular breast cancer, Mammography, Ultrasound, Magnetic resonance imaging

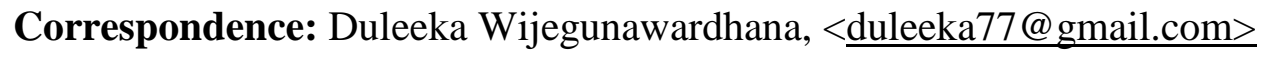

Copyright: Duleeka Wijegunawardhana

\section{Introduction}

Breast carcinoma is a heterogeneous disease accounting for $25-30 \%$ of all new cancer diagnosed in women. Invasive lobular carcinoma (ILC) is the second most common histological type accounting for $10-15 \%$ of all breast cancers. According to literature ILC is twice as common as uterine cervical cancer and is as common as ovarian cancer ${ }^{1}$. Furthermore, the incidence of ILC has been increasing over the past few decades, whilst that of invasive ductal carcinoma (IDC) has remained relatively constant. 
Clinico-pathological features of ILC

ILC of the breast has very specific immunohistopathological features differentiating it from IDC. ILC is characterised by small round cells that infiltrate the breast stroma in a single-file pattern encircling benign mammary ducts and lobules. This specific infiltration pattern of ILC through the breast tissue neither destroys the anatomic structure nor incites a desmoplastic response ${ }^{1,3}$. Hence, ILC frequently fails to form a distinct mass resulting in clinically and radiologically occult disease. Similarly, multi focal, multi centric and bilateral disease is also more common with ILC than IDC ${ }^{1,3}$. Compared with IDC, ILC is generally diagnosed at a more advanced stage with larger tumour size and more frequently with lymph node involvement. Patients diagnosed with ILCare
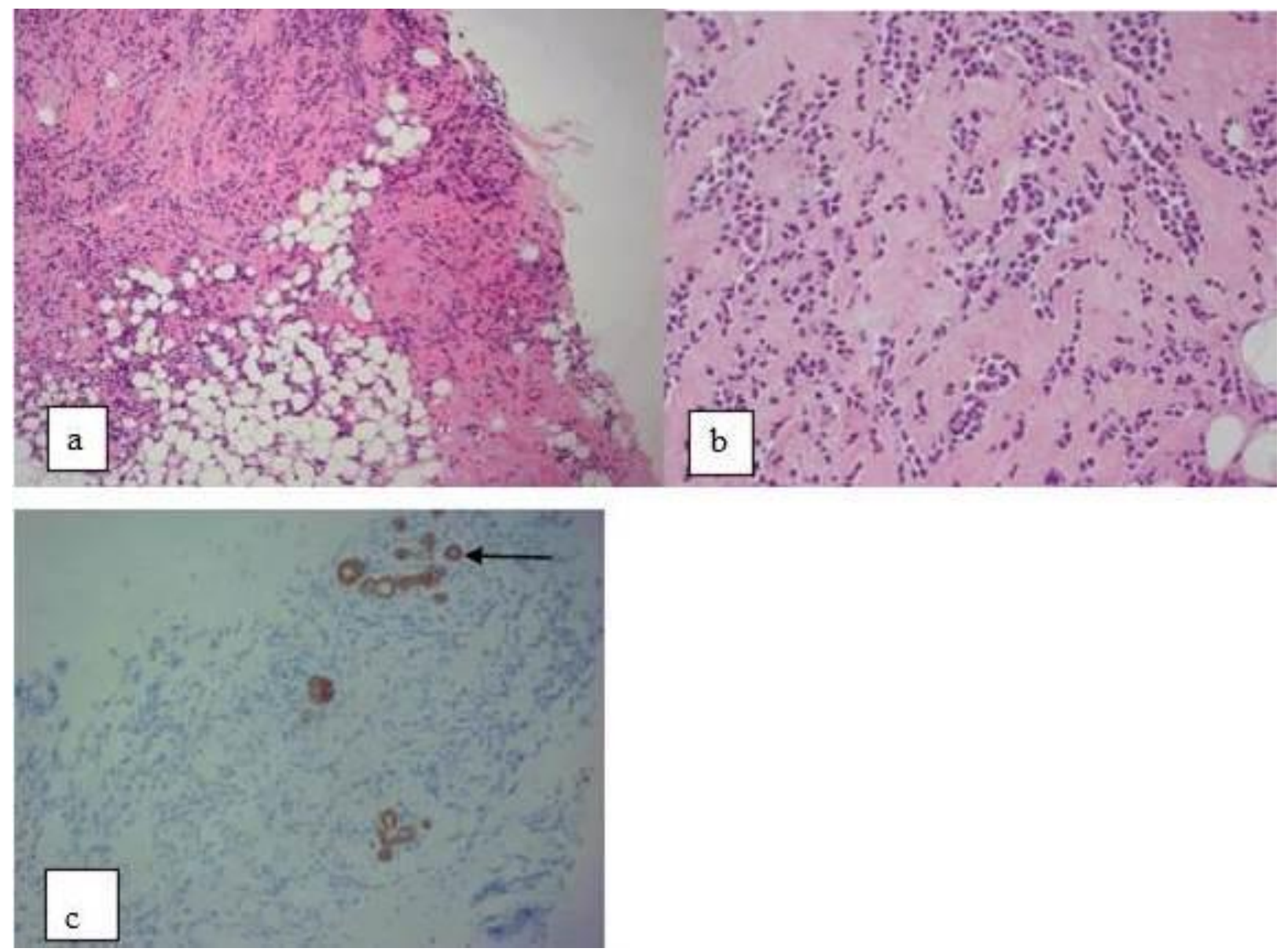

Figure 1

(a \& b) H \& E stain low and high power sections show an invasive lobular carcinoma of the breast comprising nests and chords of neoplastic cells. The classic Indian file architecture is seen. The cells are enlarged with high nuclear to cytoplasmic ratio and pleomorphic nuclei. Minimal tubule formation is observed and mitotic figures are rare amounting to an estimated grade 2.

(c) The e-cadherin expression is lost in the neoplastic cell population demonstrated by immunohistochemistry. A comparison is made with the internal control of non-neoplastic glands in the right side of the slide. $(\leftarrow)$ 
on average three years older than those with IDC. Interestingly, with all these untoward features, ILC demonstrates a more favourable stage-matched outcome compared with IDC ${ }^{3}$.

On immunohistochemistry, invasive lobular cancers are more common to have oestrogen and progesterone receptor positivity and human epidermal growth factor (HER-2),
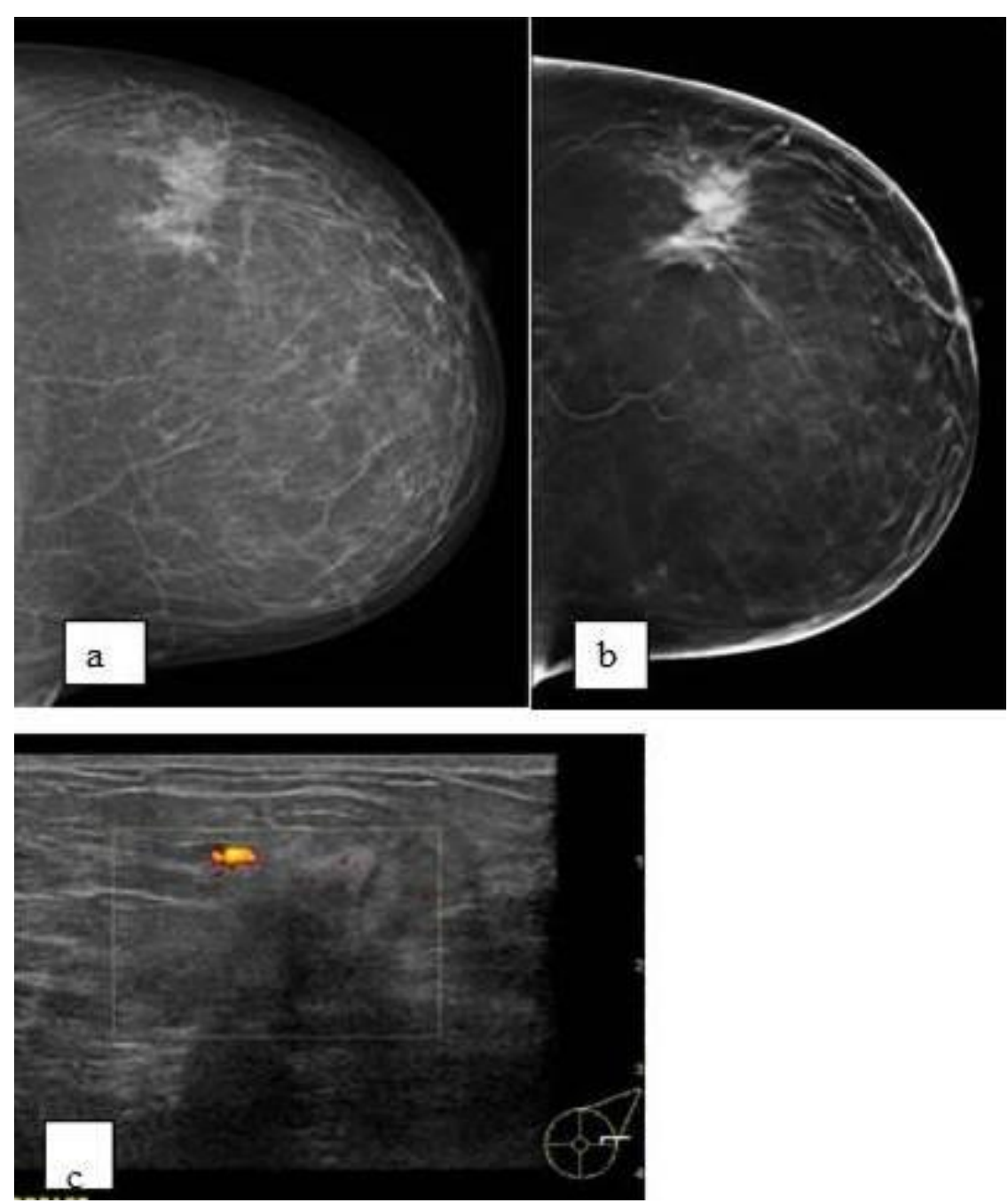

Figure 2

(a) Screening detected asymmetric density in the left lateral breast on CC mammogram.

(b) Confirmed to be a mass with spiculated margins on tomosynthesis. Vaguely palpable on clinical examination.

(c) Ultrasonography showed an ill defined, subtle focal lesion of altered echogenicity with slight increase in colour flow. Ultrasound guided biopsy confirmed invasive lobular carcinoma.
Ki67and P53 negativity than IDC. On immunohistochemistry stains lobular carcinoma shows lack of staining for Ecadherin which is the inter-cellular adhesion $\operatorname{protein}^{2,4}$ (Figure 1).

Due to high hormone receptor positivity, hormone related risk factors are thought to be strongly associated with ILC than IDC. Therefore, early menarche, late menopause, low parity, late age at first birth, oral contraceptives, hormone replacement therapy, postmenopausal obesity and alcohol consumption have all been described as potential risk factors for ILC $^{2}$. Mutations of CDH1, the gene coding for the Ecadherin are exclusively associated with ILC. BRCA2 mutations are associated with both ductal and lobular cancers, while BRCA1 and TP53 mutations are mainly associated with IDC $^{2}$.

\section{Imaging features of ILC}

Mammogram is the primary imaging tool used in breast cancer screening and in symptomatic breast disease. It is known to have a low sensitivity in detecting ILC, up to $30 \%$ being mammographically occult. Interestingly $35 \%$ of ILC 
are evident only in one mammographic projection, most commonly in cranio-caudal view $^{5}$. This low sensitivity is further compromised by dense breast tissue resulting in the mammographic sensitivity of only $11 \%$ for ILC, compared to $60 \%$ for $\mathrm{IDC}^{3}$. However, the more widespread availability of digital mammography with its superior contrast resolution over screen film imaging has contributed to some improvements in this area.

The commonest mammographic abnormality in ILC is described as a spiculated mass (Figure 2). Ill -defined mammographic masses and asymmetric densities also make a significant proportion of ILC. Architectural distortion of breast parenchyma is detected in about $20 \%$ of ILC on mammography. Compared to IDC mammographic lesions of ILC have similar or lesser density to the back ground parenchyma further contributing to its reduced visibility. Finally, microcalcifications which are readily detected on mammograms on IDC are rarely seen with $\mathrm{ILC}^{3}$.

Breast tomo synthesis is a novel development of digital mammography. It is recognised that tomo synthesis is superior to twodimensional mammography in detecting architectural distortions especially in dense breasts $^{3}$. Contrast enhanced spectral mammography (CESM) is another new imaging modality developed from digital mammography. Widely available water soluble, non ionic, low osmolar iodine contrast medium is used as the contrast agent for CESM. Once the pre contrast mammograms are subtracted from the post contrast, enhancing malignant tumour is highlighted closely resembling breast MRI (Figure 3). Principle behind this is extravasation of contrast through leaky capillaries of neo angiogenesis found in tumour tissue ${ }^{6}$. Even though this is not specific for ILC, authors found it very useful for pre-operative staging of invasive lobular carcinoma, especially in cases of subtle cancers, dense breasts and where MRI is contraindicated.

Breast ultrasonography (US) is a valuable adjunct to mammography. It confirms and further characterizes the mammographic abnormality. Moreover, US play a pivotal role in the setting of suspicious clinical examination combined with a 'normal' mammogram. Ultrasonography may detect almost $75 \%$ of the ILCs missed by mammography and represents the modality of choice for guided interventions including biopsy ${ }^{7}$.

The most common sonographic appearance of ILC is an ill-defined hypoechoic area with posterior acoustic shadowing, occurring in up to $60 \%$ of cases $^{3}$. However, ILC can merely manifest as an area of posterior acoustic shadowing without a visibly distinct mass. Presence of a dominant flow on power Doppler scan is a very promising supportive feature when dealing with subtle abnormalities. Fortunately, the overall sensitivity for the detection of ILC has been increased with the availability of high frequency transducers up to about $18 \mathrm{MHz}$. 
Magnetic Resonance Imaging of breast with intravenous Gadolinium has an overall
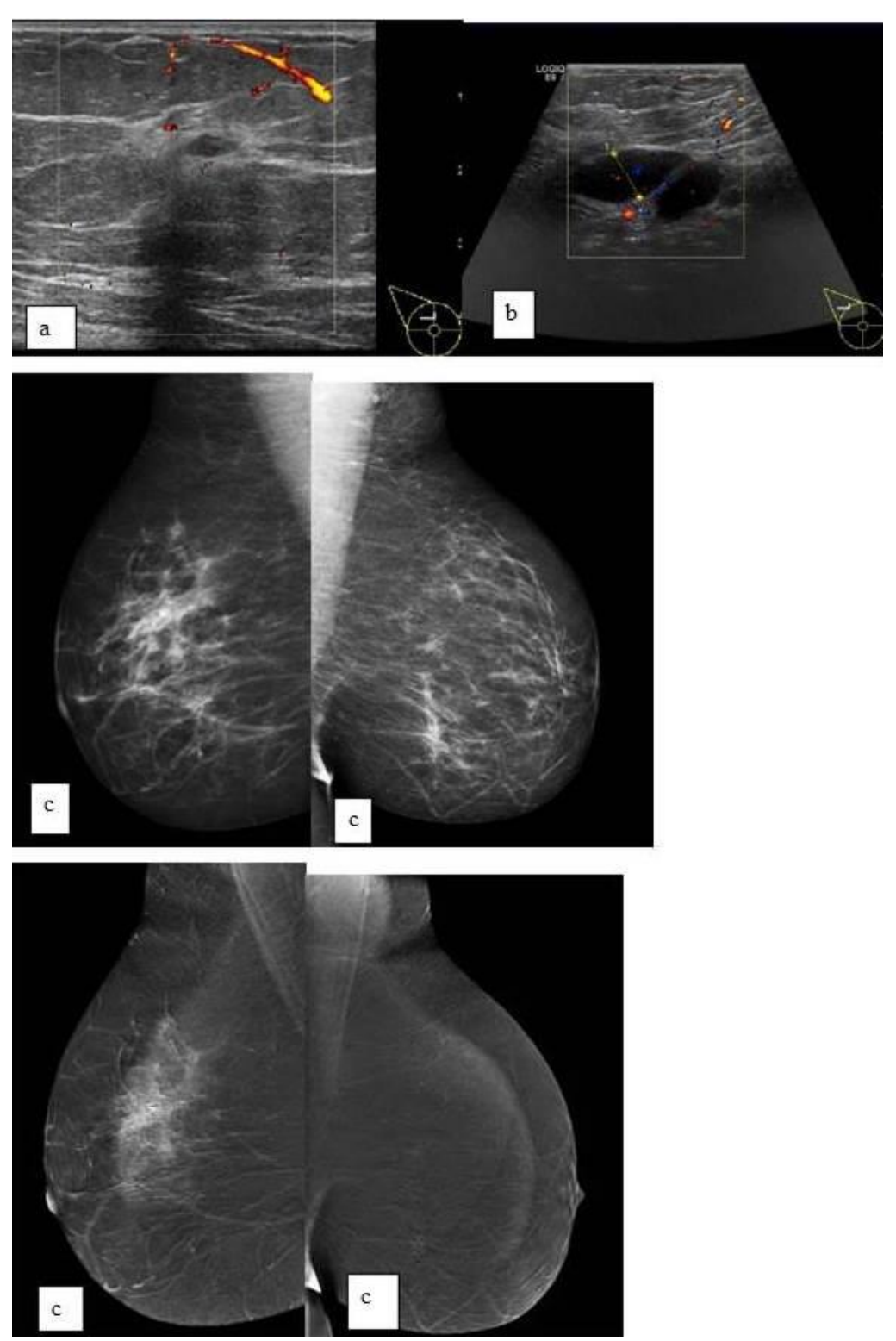

Figure 3

50 year old lady was recalled for asymmetric increased density in right upper breast on her round 1 screening mammograms.

(a) Subtle ill defined hypo-echoic area of about $15 \mathrm{~mm}$, with some shadowing and increased colour Doppler flow noted right upper outer quadrant on targeted US scan. US guided core biopsy confirmed an invasive lobular carcinoma of breast.

(b) USS demonstrating right axillary lymphadenopathy suspicious of metastasis, and confirmed on US guided FNAC

(c) Contrast enhanced spectral mammography MLO views of both breasts confirmed a larger area $(90 \mathrm{~mm})$ of abnormal enhancement in right upper breast. (similar distribution is shown on MRI) Left breast was clear of any lesions. 


\section{Review article $\mid$ SLJR}
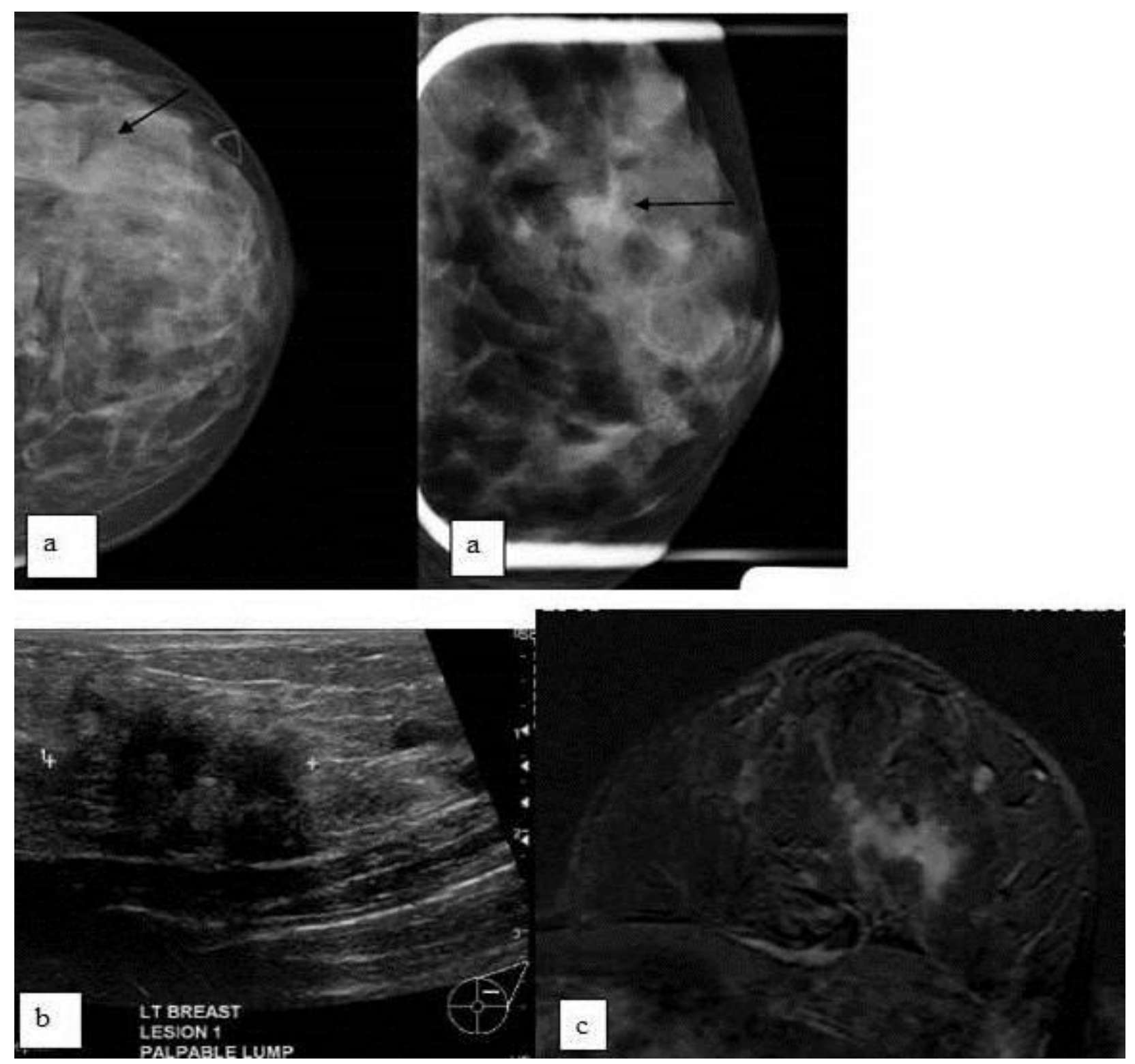

Figure 4

44 year old lady presented with a palpable lump in upper outer quadrant of left breast.

(a) Left CC and cone left MLO mammograms revealed a focal breast distortion in the area of palpable ( $\Delta$ ) abnormality, within dense breast tissue. $(\leftarrow)$

(b) Targeted US san- An ill defined hypo-echoic area with distal shadowing is noted at the palpable lump. USS guided core biopsy was reported as ILC with pleomorphic LCIS.

(c) Staging MRI Solitary enhancing mass with a few satellite nodules around it measuring larger than on other imaging. No involvement of other quadrants of left breast or right breast. 

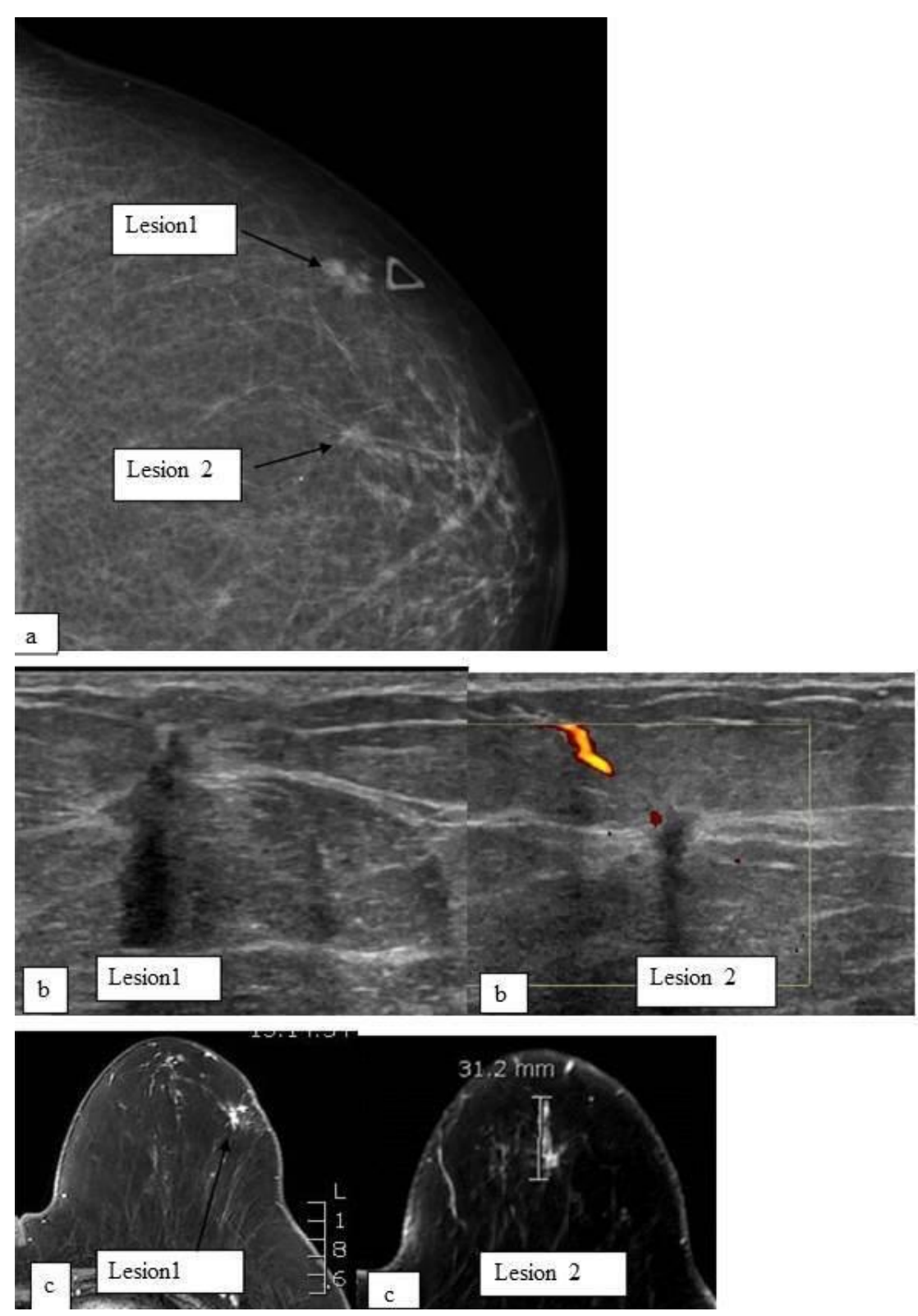

\section{Figure 5}

62 year old postmenopausal lady was recalled from breast screening for a stellate opacity in her left lateral breast. On examination, minor skin tethering was felt.

(a) Lateral half of left $\mathrm{CC}$ view revealing two small suspicious lesions within fatty breasts but only one lesion was noted left MLO view, probably due to over lapping.

(b) On US scan two small irregular hypoechoic lesions with surrounding hyper echoic halo and posterior shadowing were noted at 2 and 12'o clock positions, measuring 8 and 4 $\mathrm{mm}$ respectively. Both lesions were reported as ILC on histology following US guided core biopsy.

(c) MRI scan confirmed the two lesions in the left upper breast, with additional linear area of enhancement extending towards the nipple. Total area of abnormal enhancement measured to $60 \mathrm{~mm}$ increasing the extent of disease in the left breast. 
Recent study by Parvaiz et al. provides evidence in support of the targeted use of preoperative breast MRI for ILC rather than routine breast MRI in search of a contra lateral disease. When breast conserving surgery is contemplated for a small, uni-focal ILC on standard imaging, a breast MRI is required to exclude larger or multifocal disease $^{8-10}$. One out of every four patients with ILC have a change in their management plan due to more extensive disease detected on breast $\mathrm{MRI}^{7}$. MRI also plays a role if dense breasts preclude accurate mammographic assessment and if discrepancy is present regarding the extent of disease $^{10}$.

\section{Metastatic ILC}

Advanced primary breast cancer and axillary lymph node metastasis are relatively common at the time presentation of ILC. Distant metastasis of ILC can involve lung, liver, bone and brain similar to IDC. Apart from these recognised distant sites ILC tends to have more characteristic tumour spread to gastrointestinal tract, genitourinary tract, peritoneum, retroperitoneum and leptomeninges unlike IDC ${ }^{1}$. Higher distant metastasis rate and diffuse infiltrating metastatic process instead of a discrete nodule have also been observed in ILC compared to IDC ${ }^{1}$. For instance, metastasis to stomach results in "linitis plastica" instead of mural masses. Tiny peritoneal nodules rather than large peritoneal masses (omental cake), leptomeningeal carcinomatosis instead of cerebral metastasis and ureteric infiltration or implants are among unusual manifestations of metastatic ILC of breast ${ }^{1}$. Raap. et al. concluded that lobular breast cancer is the most common primary cancer to give rise to orbital metastases. Diffuse expansile infiltration of post septal fat causing proptosis and fusiform thickening of the extra ocular muscles often sparing globe and optic nerve have been described ${ }^{11}$.

\section{Lobular neoplasia}

Lobular neoplasia is another histological entity related to ILC. This includes atypical lobular hyperplasia and lobular carcinoma in situ on histology ${ }^{4}$. Diagnosis of a lobular neoplasia is considered as a high risk factor for development of an invasive breast cancer. They are considered as non-obligatory precursors of ILC. Lobular neoplasia is commonly found as an incidental finding on biopsy specimens. It is also represented by punctate micro calcifications on mammography and non mass enhancement on MRI.

\section{Conclusion}

Clinically and radiologically elusive nature of ILC could easily lead to false negative imaging results and delayed diagnosis. Despite the advancement of medical imaging ILC continues to pose a challenge for radiologists. Therefore, breast imaging radiologists need to be well aware of the tumour behaviour and subtle imaging characteristics of ILC to face these challenges. 


\section{References}

1. He H, Gonzalez A, Robinson E, Yang WT. Distant metastatic disease manifestations in infiltrating lobular carcinoma of the breast. American Journal of Roentgenology. 2014; 202(5):1140-8.

2. Dossus L, Benusiglio PR. Lobular breast cancer: incidence and genetic and nongenetic risk factors. Breast cancer research: BCR.2015; 17:37.

3. Johnson K, Sarma D, Hwang ES. Lobular breast cancer series: imaging. Breast cancer Research: BCR.2015; 17:94.

4. Obeng-Gyasi Samilia, Ong Cecilia, Hwang E.Shelly. Contemporary management of ductal Carcinoma in situ and lobular carcinoma in situ: Chinese clinical oncology. 2016; 5(3):32.

5. Porter AJ, Evans EB, Foxcroft LM, Simpson PT, Lakhani SR. Mammographic and radiation oncology. 2014; 58(1):1-10.

6. Luczy`nska E, Heinze-Paluchowska S, Dyczek S, et al. Contrast-enhanced spectral mammography: comparison with conventional mammography and histopathology in 152 women. Korean J Radiol 2014; 15: 689-696.

7. Oliveira TM, Elias J, Jr., Melo AF, Teixeira SR, Filho SC, Goncalves LM, et al. Evolving concepts in breast lobular neoplasia and invasive lobular carcinoma, and their impact on imaging methods. Insights into imaging.2014; 5(2):183-94.
8. Parvaiz MA, Yang P, Razia E, Mascarenhas M, Deacon C, Matey P, et al. Breast MRI in Invasive Lobular Carcinoma: A Useful Investigation in Surgical Planning?. The Breast Journal. 2016; 22(2):143-50.

9. Lau B, Romero LM. Does preoperative magnetic resonance imaging beneficially alter surgical management of invasive lobular carcinoma? The American Surgeon. 2011; 77(10):1368-71.

10. Sinclair K, Sakellariou S, Dawson N, Litherland J. Does pre operative breast MRIsignificantly impact on initial surgical procedure and re-operation rates in screen detected invasive lobular carcinoma? Clinical Radiology. 2016;71 (6):543-50.

11. Raap M, Antonopoulos W, Dammrich M, Christgen H, Steinmann D, Langer F, et al. High frequency of lobular breast cancer in distant metastases to the orbit. Cancer medicine. 2015;4 (1):104-11. 